\title{
Experimental validation of a new intensity estimation method
}

\author{
Ben Christensen (ukeben@gmail.com) \\ Derek C. Thomas \\ Kent L. Gee \\ Darren Torrie \\ Menley Stewart
}

April 16, 2014

\begin{abstract}
A new method of estimating acoustic intensity has recently been developed in an effort to improve the acoustical measurements of launch vehicles. This new method, known as the phase and amplitude gradient estimation (PAGE) method, improves upon the traditional finitedifference $p-p$ method of estimating acoustic intensity. The advantages and limitations of the PAGE method are investigated experimentally using measurements of loudspeaker arrays. The measured data is compared qualitatively to the acoustic intensity field determined by modeling the loudspeakers as baffled circular pistons using an extension of the Rayleigh integral. The primary advantage of the PAGE method is that it allows for accurate intensity measurements over a larger frequency band. When measuring smoothly varying broadband sources, it is possible to unwrap the phase component of the PAGE method, allowing for accurate intensity estimates well above previous limitations. [Work supported by NASA.]
\end{abstract}

\section{Introduction}

In this paper, we investigate the effectiveness of a new approach for the estimation of acoustic intensity, inspired by the work of Mann et al.[4] and Mann and Tichy.[2, 3] Rather than estimate the pressure gradient directly from the complex pressures, this new method uses estimates of the gradients of the pressure phase and amplitude separately. We refer to this method as the phase and amplitude gradient estimation method, or the PAGE method. This new method has been shown to be analytically superior to the traditional method of estimating acoustic intensity, the finite difference p-p method. The traditional finite difference p-p method of estimating acoustic intensity will be referred to simply as the FD method for the remainder of the paper.

A recent experiment was conducted to investigate and compare the PAGE method to the FD method. For this experiment, complicated acoustic intensity fields were created using two configurations of a loudspeaker array. First, a dipole response was created with two speaker close together with opposite phases. Second, a "tripole" source was made with three equally spaced speakers with the middle speaker having opposite phase of the outside speakers. Both these arrangements create relatively complex acoustic intensity fields. A 2D plane directly in front of the speaker array was measured using an acoustic intensity probe that was developed specifically for rocket noise measurement.

To effectively evaluate the two intensity methods we compare the measured intensities to a model of what we expect the intensity to be. Previously, the loudspeakers were modeled as point sources. This was not a sufficient model because loudspeakers only act like monopoles in the far field at low values of $k a$, where $k$ is the acoustic wavenumber, and $a$ is the radius of the loudspeakers. Representing the loudspeakers instead as baffled circular pistons provides a suitable model, and this is the focus of this work.

To determine the pressure field created by a baffled cir- 
cular piston the Rayleigh integral is commonly used.[1] By summing over the contributions of elements on the piston, an accurate complex pressure field can be predicted numerically using the Rayleigh integral. This paper will discuss extending the traditional Rayleigh integral to calculate acoustic intensity instead of only complex pressure.

\section{Theory}

\subsection{Background}

The Rayleigh integral for a surface in an infinite baffle is given by

$$
p(\mathbf{r})=\frac{j \omega \rho_{0}}{2 \pi} \int_{S} v_{n} \frac{e^{-j k R}}{R} d S
$$

where $R$ is the distance between a point on the vibrating surface and an observation point.[1] $v_{n}$ represents the velocity across the surface of the plate, which will be considered a constant for this work. This integral is used to calculate the pressure field created by a vibrating surface at any point in a half space.

For a baffled circular piston, the Rayleigh integral becomes

$$
p(\mathbf{r})=\frac{j \omega \rho_{0}}{2 \pi} \int_{0}^{2 \pi} \int_{0}^{a} v_{n} \frac{e^{-j k R}}{R} \rho d \rho d \theta
$$

where $\rho$ is the radial distance from the center of the center of the piston. As mentioned previously, the Rayleigh integral for a baffled circular pistons is often used to model loudspeakers.

\subsection{Extended Rayleigh integral}

To compute the Rayleigh integral, a surface is discretized into small pieces. Each of these pieces is assumed to radiate as a point source. The sum of the contributions from each of these point sources gives the complete pressure at any point in space. To use the Rayleigh integral to also determine the acoustic intensity, the particle velocity at any point in space must also be calculated. Since every discretized point acts like a point source, we can use the relation

$$
\vec{u}=\vec{r}\left(1-\frac{j}{k R}\right) \frac{p}{\rho_{0} c}
$$

to calculate the particle velocity using the pressure found through the Rayleigh integral. This relation depends on $\vec{r}$, which is the vector from the point source to the measurement location, and $R$ which is the magnitude of $\vec{r}$. As such, this part of the relation must be placed inside the integral since both $\vec{r}$ and $R$ vary over the surface. Plugging eq. (2) into eq. (3) results in

$$
\vec{u}=\frac{j \omega \rho_{0}}{2 \pi} \int_{S}\left(1-\frac{j}{k R}\right) \frac{\vec{r} v_{n} \frac{e^{-j k R}}{R}}{\rho_{0} c} d S .
$$

Thus the Rayleigh integral can be used to calculate the particle velocity as well as the pressure.

Equation (2) and eq. (4) can then be combine to find the active part of the acoustic intensity

$$
\vec{I}_{a}=\frac{1}{2} \Re\left\{p \vec{u}^{*}\right\} .
$$

Using this formula, we can determine the active acoustic intensity from vibrating surfaces, including the baffled circular piston.

\section{Experimental setup}

Two loudspeaker arrangements were used to create acoustic intensity fields. Both arrangements were chosen create relatively complex acoustic intensity field using a simple array of loudspeakers. The loudspeakers had a diameter of 2.5 in., and each was separated 7 in. from adjacent speakers.

First, a dipole-like field was created using two speakers with opposite phases. Second, a more complex field was generated by using three speakers in a line with the middle speaker $180^{\circ}$ out of phase with the outside speakers. Both arrangements were measured in an anechoic chamber. Perfectly coherent white noise was input into each speaker with the polarity switched on the $180^{\circ}$ out of phase speakers. Using a scanning system, a multimicrophone intensity probe was moved along a grid in front of the speaker array, and at each point in the grid the pressure at the microphones was measured. The FD and PAGE methods of estimating acoustic were applied to the pressure data at each point to find the frequency dependent intensity at each location in the grid. The result is a 2D intensity map similar to those found in the article by Mann and Tichy.[2] 
The primary advantage of the PAGE method is that it allows for accurate intensity measurements over a larger frequency band. When measuring smoothly varying broadband sources, it is possible to unwrap the phase component of the PAGE method, allowing for accurate intensity estimates well above the limitations of the FD method. This phase unwrapping is applied to the results.

To provide a comparison for the results, we must determine an expected intensity field given the source configurations. To determine the expected 2D intensity fields, we model the speakers in the array first as monopoles and then as baffled circular pistons. The previously discussed Rayleigh integral extended to calculate acoustic intensity is used to model the sources as baffled circular pistons. The measured intensity fields are compared to these models in the following section.

\section{Results}

Figures 1 to 8 seen at the end of this work compare the two models with the two methods of estimating acoustic intensity. These conclusions deal primarily as a qualitative analysis. An extensive qualitative analysis of these results will be the subject of future work.

From fig. 1 we see that at low frequencies, baffled circular pistons act much like monopoles, in that the intensity field is almost the same between the two models. Both intensity estimation methods work well at low frequencies, and match clearly match the models (see fig.2).

At higher frequencies we start to see the major differences between the two models and the two estimation methods. In fig. 3 we see that the two models differ greatly, primarily because the baffled circular piston becomes more more directional as $f$ increases, whereas the point sources are always omni-directional. Figure 4 shows that predicted intensity from the FD method has less energy than that of the PAGE method. This is because this frequency is past the limits of the FD method, and as a result, this method is no longer valid at these higher frequencies. Both the intensity magnitude and the vector directions determined by the FD method are inaccurate in this figure. The PAGE method, on the other hand, is shown to still perform well at higher frequencies, due to the unwrapped phase gradients. Comparing figs. 1 and 2 we can see clearly that the baffled circular piston is a better model for the measured loudspeaker array as the measured results clearly match this model better than the monopole model.

Similar results can be seen in figs. 5 to 8 . One interesting observation with this source configuration is that the measured intensity at $400 \mathrm{~Hz}$ (fig. 6) seems to match the monopole model better than the baffled circular piston model (fig. 5). In fig. 7 it is again seen that the PAGE method gives accurate results at higher frequencies where the FD is invalid.

\section{Conclusion}

Qualitatively, we can clearly see that the PAGE method outperforms the FD method at high frequencies. Both methods appear to work equally well at low frequencies. The PAGE method can be used passed the spatial Nyquist limit to give accurate estimates well past the limits of the FD method. Furthermore as the frequency approaches the limit of the FD method, the PAGE method does not suffer from the same frequency bias as the FD method, making the magnitudes of the PAGE method more reliable.

It was also shown that the acoustic intensity of a baffled circular piston can be calculated by extending the Rayleigh integral to also calculate particle velocity. Modeling loudspeakers as baffled circular pistons is seen to be more accurate than modeling them as point sources.

Future work will include conducting a rigorous quantitate comparison of the measured intensity fields to the models, whereas this analysis has been primarily qualitative. A new data set will also be taken to correct errors due to a mis-calibrated scanning system.

\section{References}

[1] Frank J Fahy and Paolo Gardonio. Sound and structural vibration: radiation, transmission and response. Academic press, 2007.

[2] J. Adin Mann III and Jiri Tichy. Acoustic intensity analysis: Distinguishing energy propagation and wave-front propagation. The Journal of the Acoustical Society of America, 90(1):20-25, 1991. 
[3] J. Adin Mann III and Jiri Tichy. Near-field identification of vibration sources, resonant cavities, and diffraction using acoustic intensity measurements. The Journal of the Acoustical Society of America, 90(2):720-729, 1991.

[4] J. Adin Mann III, Jiri Tichy, and Anthony J. Romano. Instantaneous and time-averaged energy transfer in acoustic fields. The Journal of the Acoustical Society of America, 82(1):17-30, 1987.

[5] Derek C Thomas. Theory and estimation of acoustic intensity and energy density. $\mathrm{PhD}$ thesis, Brigham Young University. Department of Physics and Astronomy, 2008. 

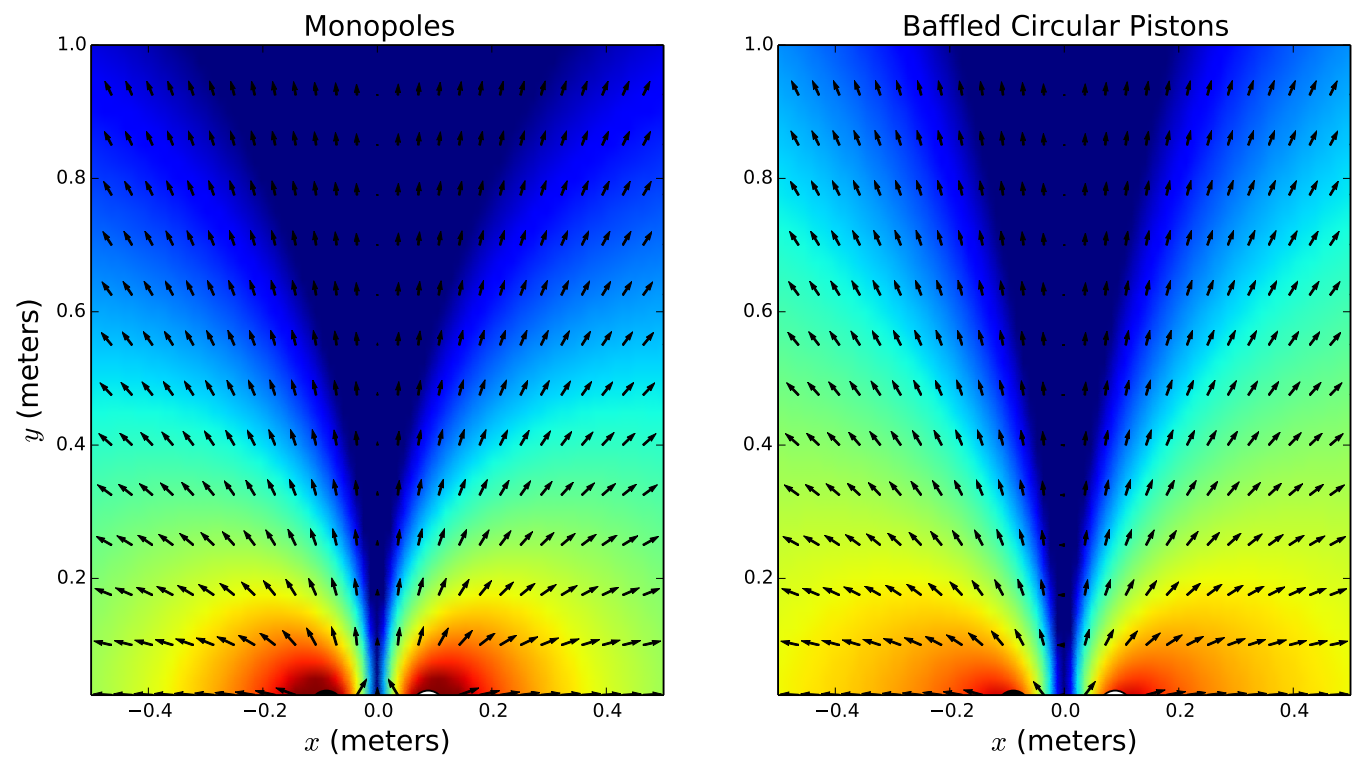

Figure 1: The modeled acoustic intensity field from two closely spaced, of phase speakers at $400 \mathrm{~Hz}$. Depicted is both the monopole (left) and baffled circular piston (right) models. 

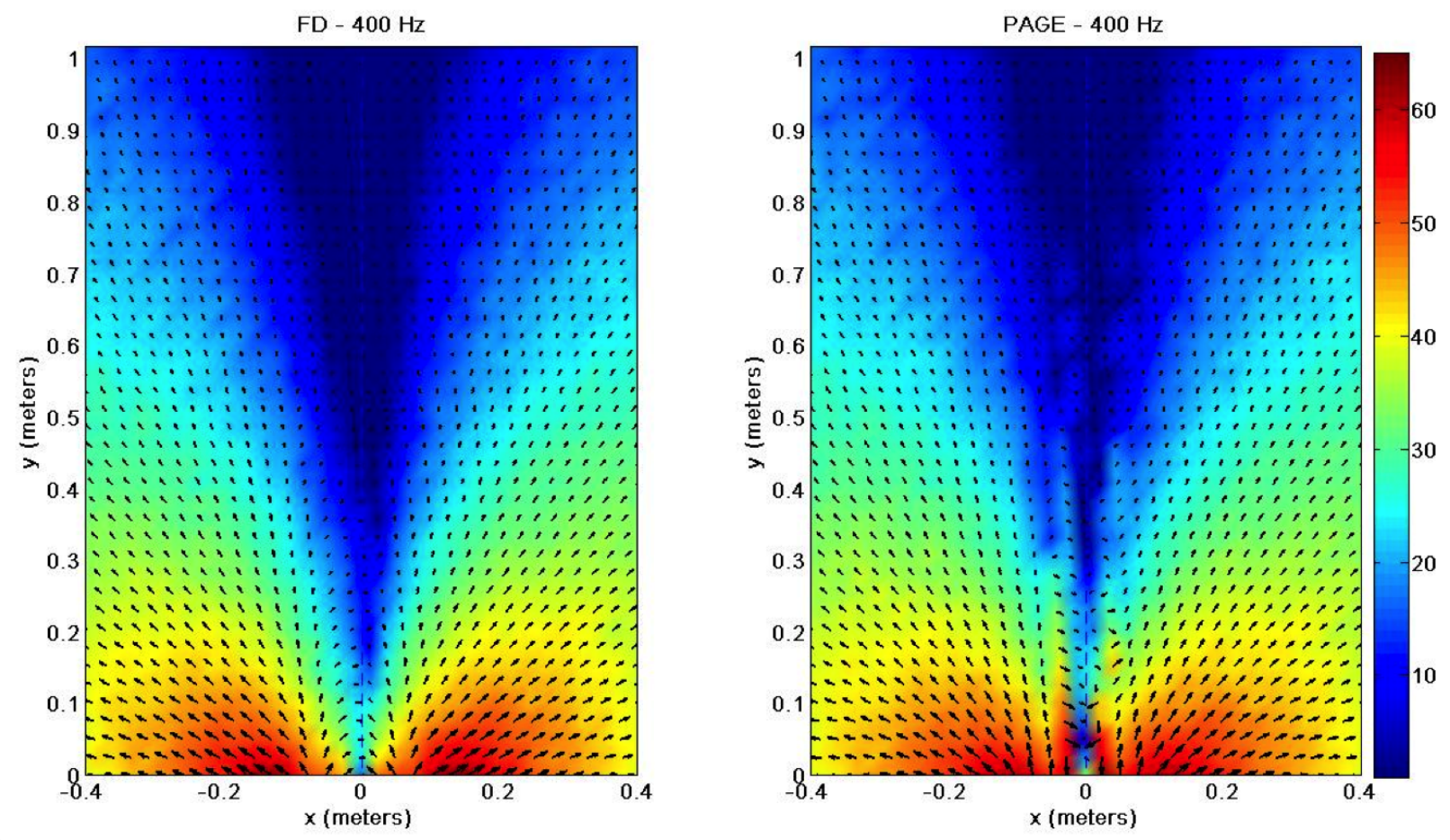

Figure 2: The measured acoustic intensity field from two closely spaced of phase speakers at $400 \mathrm{~Hz}$. Each vector position represents a measurement location. Intensity was processed using both the FD (left) and PAGE (right) methods. 

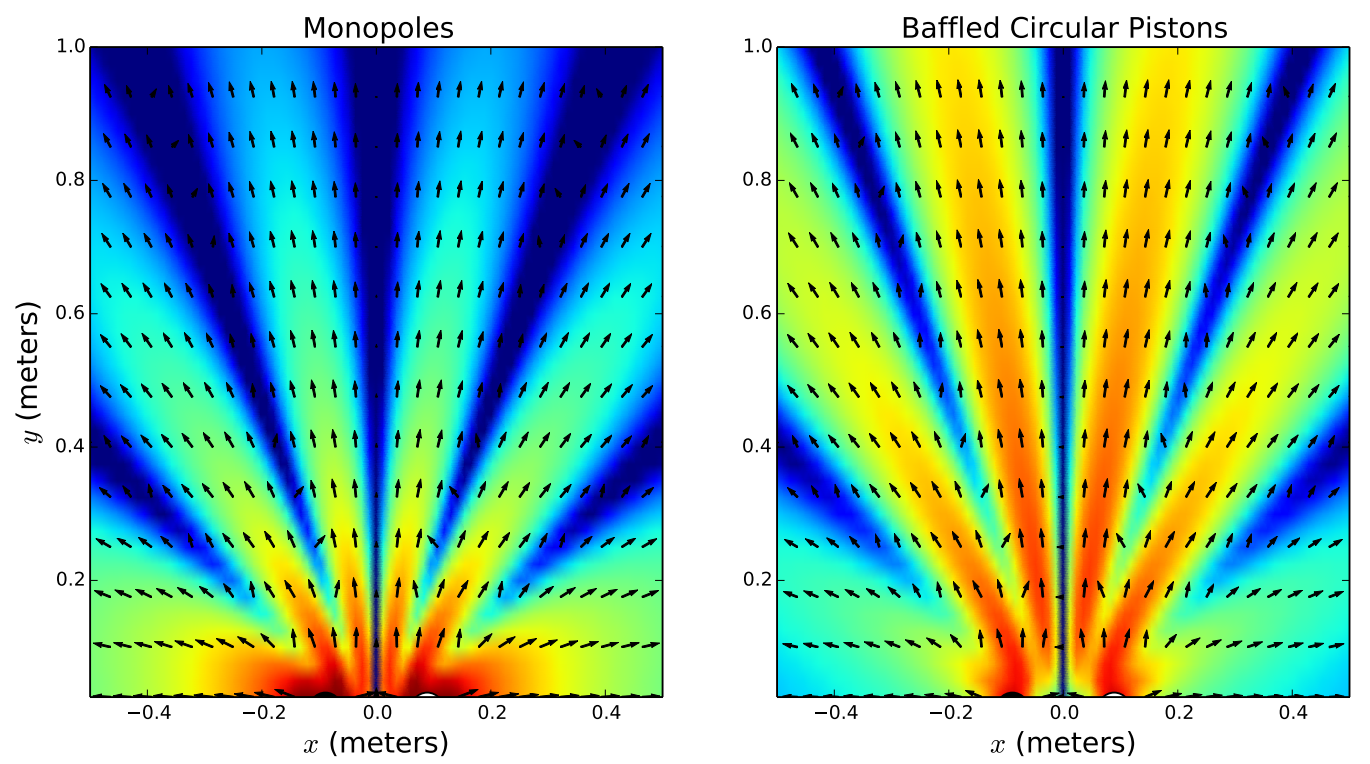

Figure 3: The modeled acoustic intensity field from two closely spaced, of phase speakers at $5000 \mathrm{~Hz}$. Depicted is both the monopole (left) and baffled circular piston (right) models. 

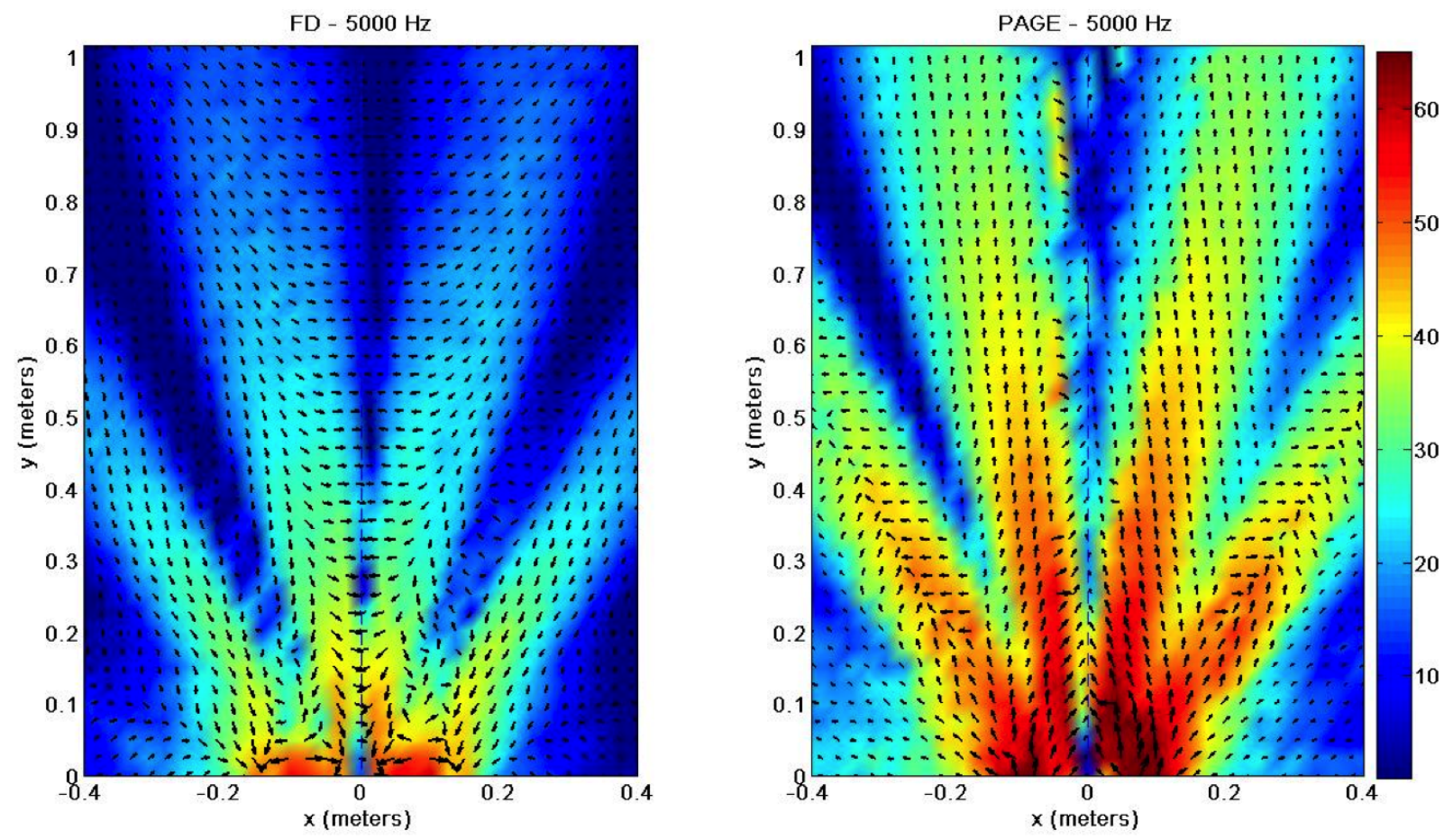

Figure 4: The measured acoustic intensity field from two closely spaced of phase speakers at $5000 \mathrm{~Hz}$. Each vector position represents a measurement location. Intensity was processed using both the FD (left) and PAGE (right) methods. 

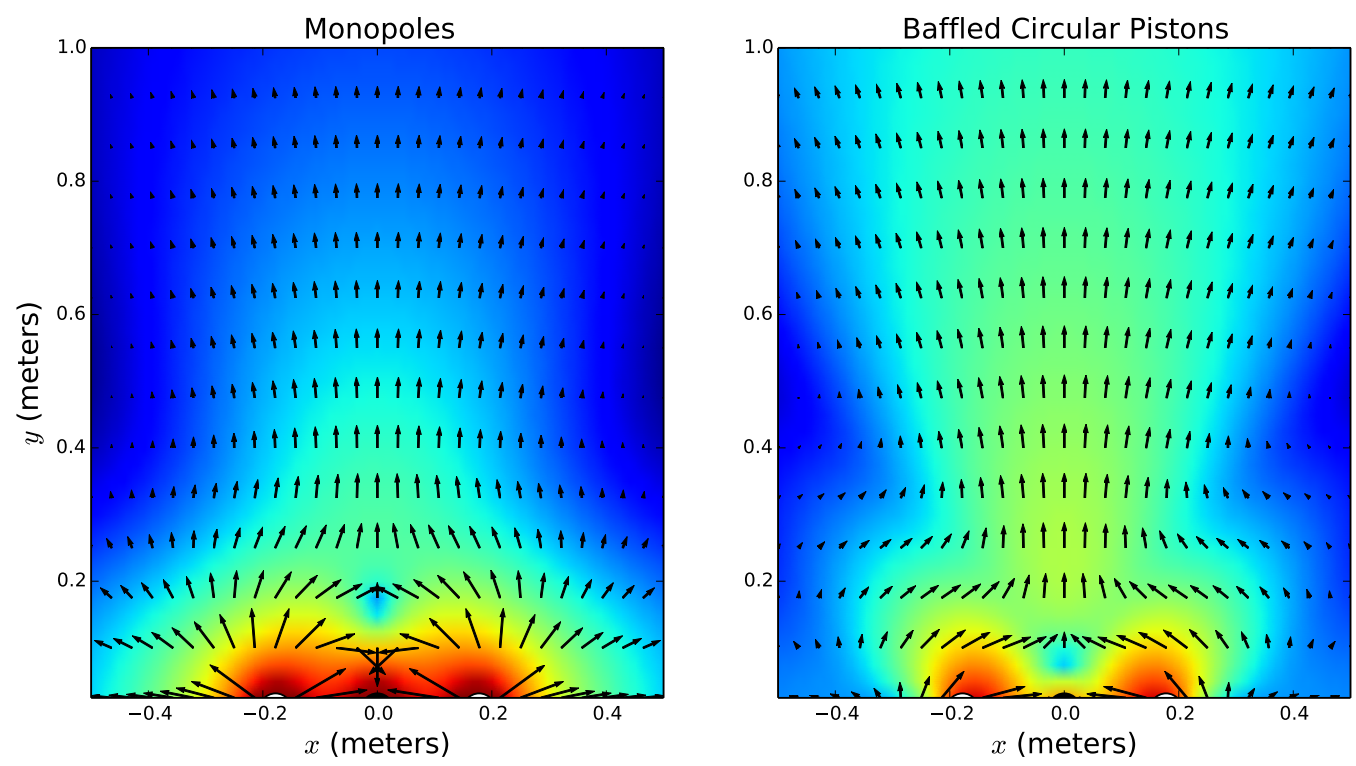

Figure 5: The modeled acoustic intensity field from three closely spaced speakers with the middle speaker out of phase with the outside speakers, at $400 \mathrm{~Hz}$. Depicted is both the monopole (left) and baffled circular piston (right) models. 

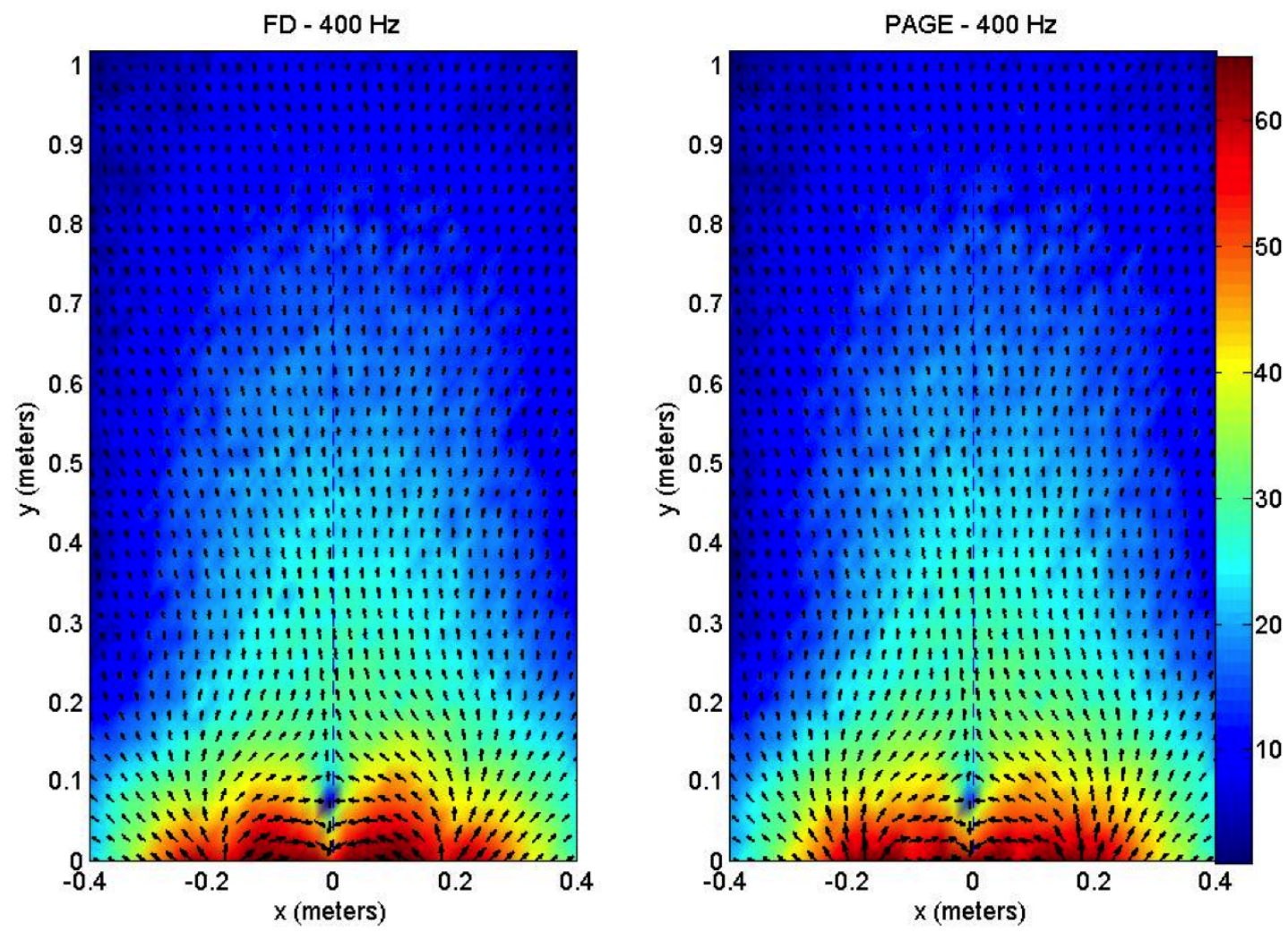

Figure 6: The measured acoustic intensity field from three closely spaced speakers with the middle speaker out of phase with the outside speakers, at $400 \mathrm{~Hz}$. Each vector position represents a measurement location. Intensity was processed using both the FD (left) and PAGE (right) methods. 

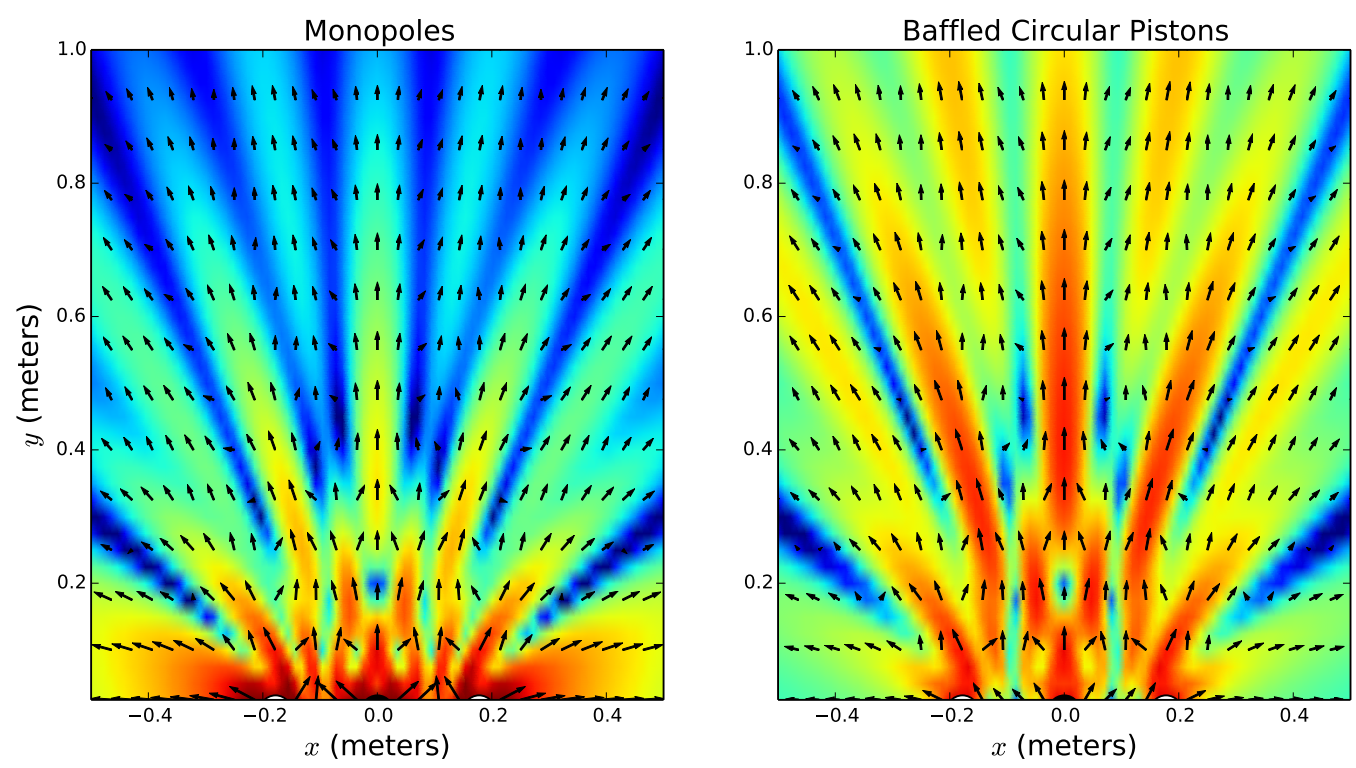

Figure 7: The modeled acoustic intensity field from three closely spaced speakers with the middle speaker out of phase with the outside speakers, at $5000 \mathrm{~Hz}$. Depicted is both the monopole (left) and baffled circular piston (right) models. 

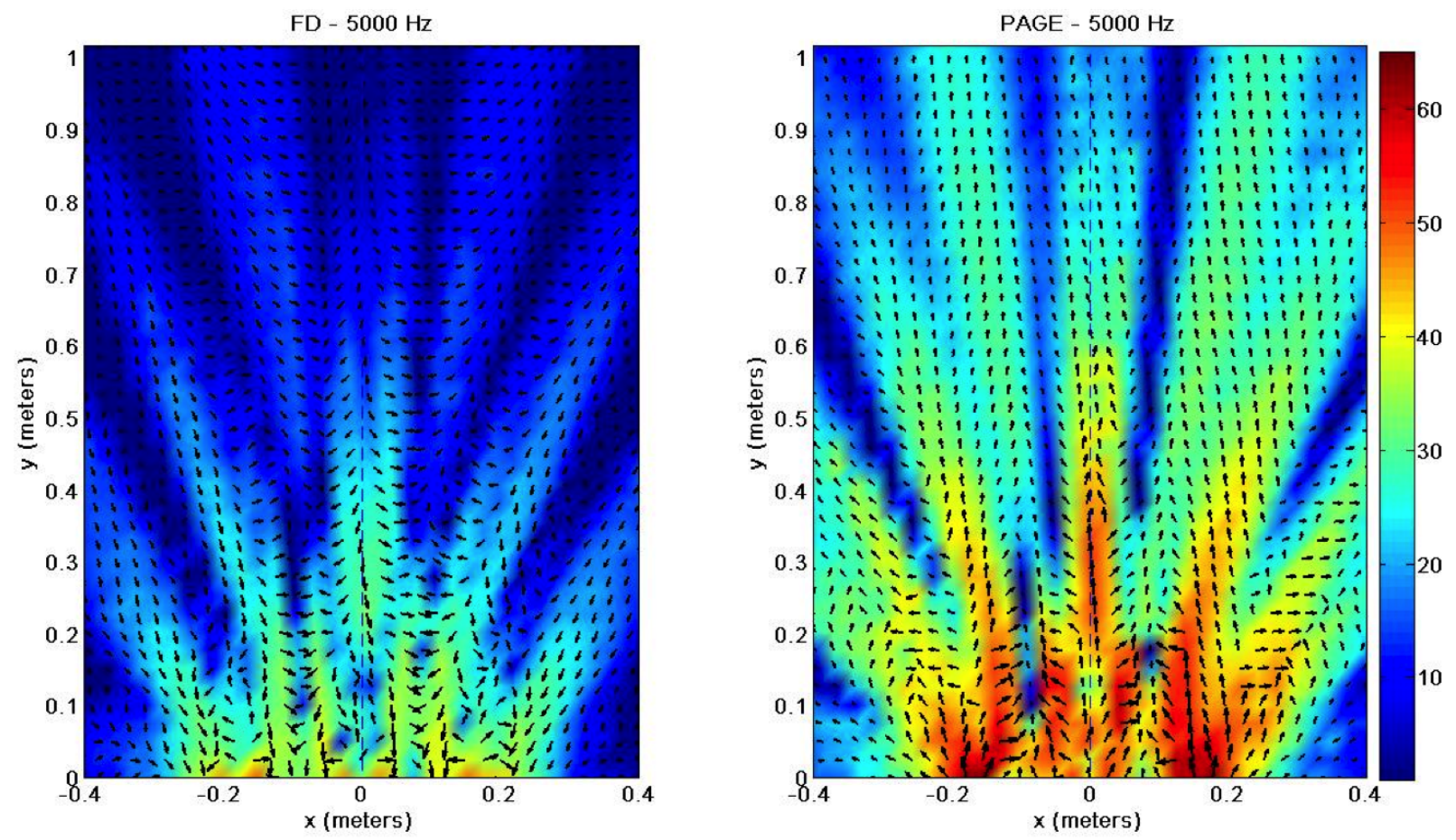

Figure 8: The measured acoustic intensity field from three closely spaced speakers with the middle speaker out of phase with the outside speakers, at $5000 \mathrm{~Hz}$. Each vector position represents a measurement location. Intensity was processed using both the FD (left) and PAGE (right) methods. 